\title{
Bone density of first and second segments of normal and dysmorphic sacra
}

\author{
Joseph M. Radley ${ }^{1^{*}} \mathbb{0}$, Brian W. Hill², Daemeon A. Nicolaou², Stephen B. Huebner ${ }^{3}$, Kelby B. Napier ${ }^{4}$ \\ and Dane H. Salazar ${ }^{5}$
}

\begin{abstract}
Background: Iliosacral screw fixation is safe and effective but can be complicated by loss of fixation, particularly in patients with osteopenic bone. Sacral morphology dictates where iliosacral screws may be placed when stabilizing pelvic ring injuries. In dysmorphic sacra, the safe osseous corridor of the upper sacral segment (S1) is smaller and lacks a transsacral corridor, increasing the need for fixation in the second sacral segment (S2). Previous evidence suggests that S2 is less dense than S1. The aim of this cross-sectional study is to further evaluate bone mineral density (BMD) of the S1 and S2 iliosacral osseous pathways through morphology stratification into normal and dysmorphic sacra.

Materials and methods: Pelvic computed tomography scans of 50 consecutive trauma patients, aged 18 to 50 years, from a level 1 trauma center were analyzed prospectively. Five radiographic features (upper sacral segment not recessed in the pelvis, mammillary bodies, acute alar slope, residual S1 disk, and misshapen sacral foramen) were used to identify dysmorphic characteristics, and sacra with four or five features were classified as dysmorphic. Hounsfield unit values were used to estimate the regional BMD of S1 and S2. Student's $t$-test was utilized to compare the mean values at each segment, with statistical significance being set at $p<0.05$. No change in clinical management occurred as a result of inclusion in this study.
\end{abstract}

Results: A statistical difference in BMD was appreciated between S1 and S2 in both normal and dysmorphic sacra $(p<0.0001)$, with $28.4 \%$ lower density in S2 than S1. Further, S1 in dysmorphic sacra tended to be $4 \%$ less dense than $\mathrm{S} 1$ in normal sacra $(p=0.047)$. No difference in density was appreciated at $\mathrm{S} 2$ based on morphology.

Conclusions: Our results would indicate that, based on BMD alone, fixation should be maximized in S1 prior to fixation in S2. In cases where S2 fixation is required, we recommend that transsacral fixation should be strongly considered if possible to bypass the $\$ 2$ body and achieve fixation in the cortical bone of the ilium and sacrum.

Level of evidence: Level III.

Keywords: Iliosacral screws, Pelvic fracture fixation, Pelvic ring disruptions, Sacral dysmorphism, Regional bone density

\section{Introduction}

Osseous fixation pathways within the pelvis have been well described [1]. The iliosacral screw corridor has been increasingly utilized for management of pelvic ring injuries [2-8]. The technique for implantation of iliosacral

\footnotetext{
*Correspondence: radleyjm1@gmail.com

1 Department of Orthopaedic Surgery, University of Pittsburgh Medical

Center-Hamot, 201 State Street, Erie, PA 16550, USA

Full list of author information is available at the end of the article
}

screws has been shown to be safe and effective when performed properly [5-18]. When employed with closed reduction and percutaneous insertion, this technique can rapidly stabilize the pelvis with minimal morbidity for the patient [2-8]. However, placement of iliosacral screws requires a detailed understanding of sacral anatomy. Previous anatomic studies revealed an anatomic variation that differs from the "normal" phenotype. This "dysmorphic" variant has anatomic restraints that limit iliosacral fixation into the first sacral segment (S1), while being 
more open to fixation in the second sacral segment (S2) [15-18].

While screw insertion into both S1 and S2 has been shown to be safe, little has been written on the bone density of each sacral segment. Computed tomography (CT) scan has attracted interest as a means of evaluating bone mineral density (BMD) from studies ordered for other diagnostic purposes. Recent studies have evaluated the application of this technique to the sacrum [19, 20]. Zou et al. compared CT-acquired Hounsfield unit (HU) values at $\mathrm{S} 1$ with validated gold-standard DEXA and CT at L1, establishing guidelines for the use of $\mathrm{HU}$ in the sacrum as a marker for osteoporosis [19]. Salazar et al. [20] studied otherwise healthy trauma patients showing relative osteopenia of S2 in comparison with S1, which may have implications for iliosacral screw fixation of pelvic ring injuries.

Iliosacral screw fixation failure has been described in literature associated with osteopenic bone $[6,21]$. The purpose of this study is to expand upon the understanding of regional BMD of the sacrum in otherwise healthy trauma patients through stratification of sacra based upon morphology. Using CT-scan HU values, we reexamined the difference in bone density at S1 compared with S2 in both normal and dysmorphic sacra. Further, we examine whether there is a difference in density between normal and dysmorphic sacra at each level. Based upon clinical observations, we hypothesize that, in normal and dysmorphic sacra, there will be a relative osteopenia of S2 compared with S1. We also hypothesize that lower density will be found in the dysmorphic sacra compared with the normal phenotype.

\section{Materials and methods}

This study was approved by our institutional review board. Pelvic CT scans of 50 consecutive patients between the ages of 18 to 50 years were prospectively evaluated. Patient care was not altered as a result of this study. CT data used were collected as a routine component of trauma workup. CT scans were excluded for previous documented sacral trauma, lumbar/sacral implants, sacral fracture, neoplasm of the pelvic girdle, rheumatoid arthritis, seronegative arthropathies, osteoporosis/osteopenia, paraplegia, nonambulatory/wheelchair bound status, or signs of malnutrition. Patients were also excluded for use of bisphosphonates, steroids, or hormonal medications. Exclusion criteria for inadequate scan technique limiting density determination included motion artifact, streak artifact, beam hardening artifact, or photon deprivation in the extremely obese patient. The subjects' age and gender were recorded.

After identification of the patient's CT scans, two musculoskeletal radiologists independently identified features of dysmorphic sacra. For the purposes of this study, these features included: (1) an upper segment that is not recessed in the pelvis, (2) the presence of mammillary bodies, (3) an acute alar slope, (4) a residual disc between the first and second sacral segments, and (5) noncircular upper sacral neural foramina (Fig. 1). These features were chosen based on prior study on sacral morphology with

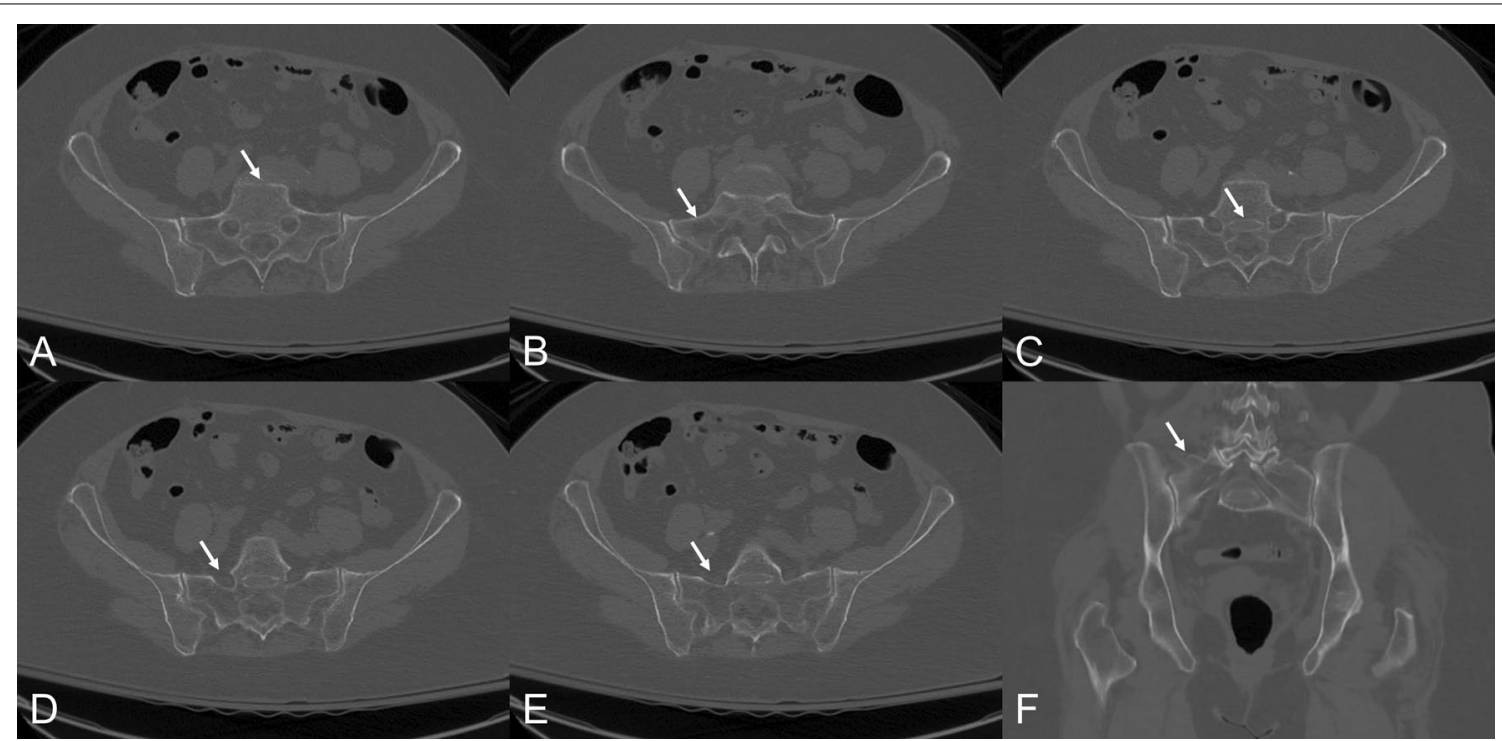

Fig. 1 CT images showing dysmorphic features. Axial images demonstrate: (a) S1 not recessed in the pelvis, (b) acute alar slope, (c) residual disk, and $(\mathbf{d}, \mathbf{e})$ misshapen sacral foramina. Coronal imaging demonstrates (f) mammillary bodies 
regard to sacral dysmorphism [15-18]. Sacra with four or five features were classified as dysmorphic.

A Hounsfield unit value for each sacral segment was then calculated adapting the methodology from Salazar et al. [20]. For each sacral segment, four circular regions of interest (ROIs) were strategically placed utilizing the axial CT sections (Fig. 2). The ROIs were placed into the anterior, posterior, right lateral, and left lateral aspects of each sacral body utilizing the axial CT imaging via the picture archiving and communication system (PACS) software. These were positioned to minimize overlap among the individual ROIs. Once placed, a $\mathrm{HU}$ value was obtained for each ROI. The four values were then averaged to arrive at a single $\mathrm{HU}$ value for each sacral segment.

\section{Statistical analysis}

Prospective power analysis revealed that a sample size of 25 patients was necessary to detect a difference in S1 compared with S2 at the 0.05 alpha level with $80 \%$ power. The data collected were analyzed utilizing SPSS 25.0 (IBM Corp) statistical software. The variables were tested for normal distribution, and the data were not skewed. Student's $t$-test was utilized to compare the mean HU values at each segment, with statistical significance being set at $p<0.05$. Mann-Whitney $U$-test with chi-squared comparison was used to assess dysmorphic sacra. Interrater reliability was performed utilizing percent agreement and Cohen's kappa statistics.

\section{Results}

Two CT scans were excluded after radiographic analysis due to inadequate imaging and repeated imaging of another subject with a different electronic medical record number. The remaining 48 patients whose $\mathrm{CT}$ scans were analyzed had a mean age of 33.7 years ( $18-50$ years). The majority of the subjects were male $(39 / 48,81 \%)$.

The mean HU for S1 (320.9 HU, 204.25-447.25 HU) was significantly greater than that for S2 $(229.8 \mathrm{HU}$, $107.37-408.6 \mathrm{HU})(p<0.0001)$ (Table 1). Subgroup analysis showed that this was true in both normal sacra (S1: $323 \mathrm{HU}$ versus S2: $234 \mathrm{HU} ;<0.001$ ) and dysmorphic sacra (four or more dysmorphic features; S1: $320 \mathrm{HU}$ versus S2: $228 \mathrm{HU} ; p<0.0001$ ). With respect to the S1 body, the mean $\mathrm{HU}$ at the anterior $(p=0.002)$, right lateral $(p<0.001)$, and left lateral $(p=0.0017)$ ROIs were significantly greater than that at the posterior ROI. For the S2 body, the mean $\mathrm{HU}$ of the anterior ROI was significantly $(p=0.003)$ greater than those of the right lateral, left lateral, and posterior ROIs.

Further evaluation with regard to anatomical variance showed that $35 / 48(72.9 \%)$ of the pelvises were identified as having at least one dysmorphic feature by the musculoskeletal radiologists (Table 2). Of the patients sampled,

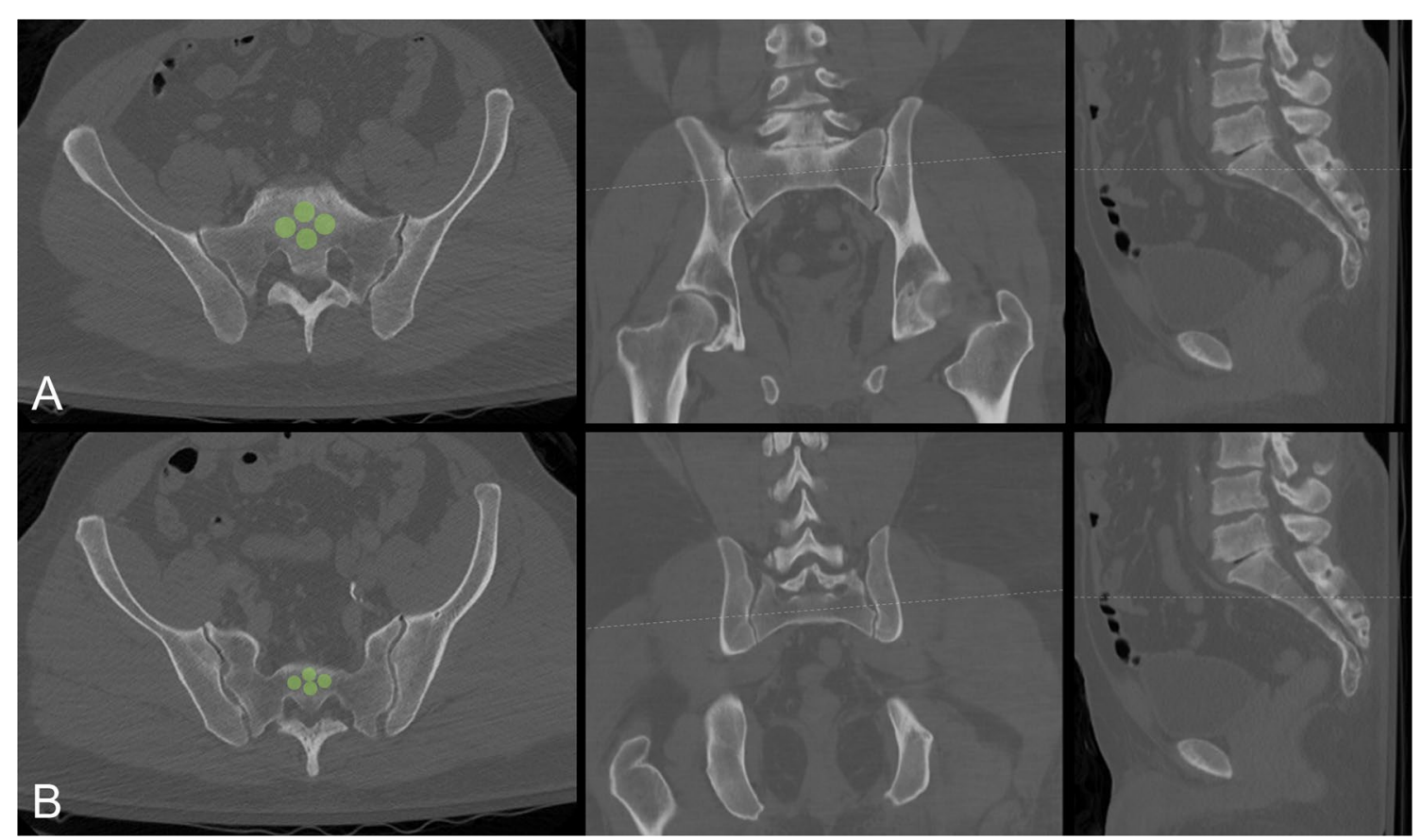

Fig. 2 Axial, sagittal, and coronal CT images depicting cross-referencing technique used for localization of regions of interest (ROIs) represented by green circles in (a) S1 and (b) S2 
Table 1 Demographics and mean HU measures

\begin{tabular}{ll}
\hline Subjects & 48 \\
Age (years) & $33.7(18-50)$ \\
Gender & $39 \mathrm{M}, 9 \mathrm{~F}$ \\
Mean S1 (HU) & $320.9^{\dagger}$ \\
S1 anterior (HU) & 329 \\
S1 right $(\mathrm{HU})$ & 333 \\
S1 left $(\mathrm{HU})$ & 332 \\
S1 posterior $(\mathrm{HU})$ & 287 \\
Mean S2 (HU) & $229.8^{\dagger}$ \\
S2 anterior (HU) & 253 \\
S2 right $(\mathrm{HU})$ & 229 \\
S2 left $(\mathrm{HU})$ & 218 \\
S2 posterior (HU) & 217 \\
$\leq 3$ dysmorphic features & \\
S1 (HU) & $326^{\S}$ \\
S2 (HU) & 230 \\
$>3$ dysmorphic features & \\
S1 (HU) & $313^{\S}$ \\
S2 (HU) & 225 \\
\hline
\end{tabular}

HU Hounsfield units

$+p<0.0001$

$\S p=0.047$

Table 2 Prevalence of dysmorphic sacral features

\begin{tabular}{lc}
\hline Upper sacral segment not recessed in the pelvis & $10.4 \%$ \\
Mammillary bodies & $18.8 \%$ \\
Acute alar slope & $12.5 \%$ \\
Residual disk & $70.8 \%$ \\
Misshapen sacral foramen & $12.5 \%$ \\
Dysmorphic features & \\
0 & $27.08 \%$ \\
1 & $50.0 \%$ \\
2 & $6.25 \%$ \\
3 & $6.25 \%$ \\
4 & $4.17 \%$ \\
5 & $6.25 \%$ \\
\hline
\end{tabular}

$5 / 48(10.4 \%)$ had an upper sacral segment not recessed in the pelvis, $9 / 48(18.8 \%)$ had mammillary bodies, $6 / 48$ (12.5\%) had acute alar slope, $34 / 48$ (70.8\%) had a residual disk, and 6/48 (12.5\%) had misshapen sacral foramen. Thirteen $(27 \%)$ were identified as having no dysmorphic features. Twenty-four (50\%) were identified as having one characteristic, 3/48 (6\%) as having two characteristics, $3 / 48(6 \%)$ as having three characteristics, $2 / 48(4 \%)$ as having four characteristics, and 3/48 (6\%) as having all five characteristics. When there were four or more dysmorphic features identified, the mean $\mathrm{HU}$ at S1 tended to be less than that of subjects with three or fewer dysmorphic features (313 HU versus $326 \mathrm{HU}$, respectively; $p=0.047)$. There were no HU differences at the S2 body regardless of the number of dysmorphic features.

The interrater reliability between the two musculoskeletal radiologists showed substantial to excellent agreement for four of the five dysmorphic features. Cohen's kappa failed to show such agreement for the residual S1 disk (Table 3).

\section{Discussion}

We confirmed our first hypothesis showing that the average density of S2 was $28.4 \%$ lower than S1. A subgroup analysis showed similar results. These findings corroborate the earlier results by Salazar et al. [20] in a study of 25 normal sacra in which S2 was $28.1 \%$ less dense. Further analysis of our results showed that density was highest in the anterior and lateral ROIs of S1. Although the $\mathrm{ROI}$ in the posterior aspect of S1 was found to have a lower density than the remainder of S1, this was found to have higher density than all aspects of S2.

Our second hypothesis that dysmorphic sacra would have lower BMD was confirmed in S1 but not in S2. Our study results show that $\mathrm{S} 1$ in dysmorphic sacra tended to have lower density than the normal morphology. The explanation for this difference is not elucidated by our dataset, but we believe that differences in anatomy may alter the biomechanics of force transmission during weight bearing and thus density based upon Wolff's law. Further study on the biomechanics of force transmission in normal versus dysmorphic sacra would be needed to confirm this; however, while statistically significant, only a $4 \%$ decrease was found, which may lack clinical significance in regards to biomechanical impact on fixation strength. S2 showed no difference in density based on morphology, which may be a result of more anatomic similarity at S2 compared with S1.

Prior biomechanical studies on pelvic ring injuries have shown improved stability with multiple iliosacral screws $[22,23]$. Our results would support that the most dense bone for fixation would be the anterior aspect of $\mathrm{S} 1$, followed by the posterior aspect of S1, when possible.

Table 3 Interrater reliability

\begin{tabular}{lll}
\hline & Agreement (\%) & Kappa value \\
\hline $\begin{array}{l}\text { Upper sacral segment not } \\
\text { recessed in the pelvis }\end{array}$ & 71 & $0.810-0.911$ \\
Mammillary bodies & 67 & $0.606-0.650$ \\
Acute alar slope & 67 & $0.704-0.765$ \\
Residual disk & 81 & $0.104-0.829$ \\
Misshapen sacral foramen & 55 & $0.592-0.728$ \\
\hline
\end{tabular}


Unfortunately, the S1 anatomy is not always amenable to multiple points of fixation, in particular in sacra with dysmorphic features. In these sacra, the $\mathrm{S} 1$ corridor is $36 \%$ smaller and typically lacks a safe transsacral S1 corridor. The S2 corridor is generally more open to fixation, with roughly twice the cross-sectional area. This limits iliosacral fixation options in S1 and encourages fixation into the less dense $\mathrm{S} 2[17,18]$.

Sacral dysmorphism has been reported in $41-44 \%$ of the population $[17,18]$. This reported prevalence is higher than in our experimental patient set (10.42\%), using greater than three features for identification. To our knowledge, there is no specific criteria for designation of a sacrum as dysmorphic. Gardner et al. [17] used the overall appearance of the sacrum rather than a specific number of features for identification. Our data show a significant diversity of sacral anatomy based on identification of dysmorphic features. We believe that this highlights a need to place less emphasis on defining dysmorphism by specific radiographic features and more emphasis on the clinically relevant S1 osseous corridor anatomy.

For this study, we utilized opportunistically obtained CT scans during the initial trauma assessment. CT scan has been shown to be a powerful tool in evaluating bone density. Early studies comparing DEXA with quantitative CT scans demonstrated that CT was capable of accurately estimating regional cancellous bone mineral density [24, 25]. More recent studies have assessed bone mineral density utilizing CT scans obtained for other diagnostic reasons [19, 20, 26-29]. Zou et al. showed that $\mathrm{HU}$ values obtained from CT scans of the sacrum can be effectively used to assist in diagnosis of osteoporosis. Setting a cutoff value of $222 \mathrm{HUs}$ at S1, this was a $90 \%$ sensitive test for the diagnosis of osteoporosis [19]. Diagnosis of osteoporosis in this study was based on DEXA and $\mathrm{HU}$ values at L1, which has been validated by Hoel et al. [29]. Our data would indicate that the bone present in the body of S2, averaging between 225 and $230 \mathrm{HUs}$, is only slightly more dense than the proposed 222 HUs cutoff for osteoporosis in $\mathrm{S} 1$. This raises concerns about the quality of bone available for fixation in S2 when fixation in S1 is limited.

There are limitations to this study. The patient population was predominantly young and male, which limits the application of these findings broadly. Despite this, iliosacral screw fixation is more commonly performed in young men. Thus, the specific study of a young male population strengthens the application of these findings to the realistic environment of trauma centers.

There are also limitations in using $\mathrm{CT}$ as a measure for regional bone density. While CT is able to delineate quantitative bone mineral density, this does not provide a qualitative measure of the physical cancellous microarchitecture. Invasive bone biopsy would be necessary to evaluate this.

This study reaffirms a relative osteopenia of S2 in comparison with S1, regardless of sacral morphology. The clinical significance of this difference would require biomechanical study. However, this raises concerns in particular for the management of unstable pelvic ring injury in patients with dysmorphic sacra. The limited fixation options in S1 lead to the increased need for fixation in the less-dense S2. We would argue that, in general, fixation should be maximized in S1 prior to fixation in S2, based on bone mineral density. In cases where fixation in S2 is utilized, strong consideration should be given to transsacral fixation, where fixation is achieved in the far-sided cortical bone rather than in the less-dense cancellous bone of S2.

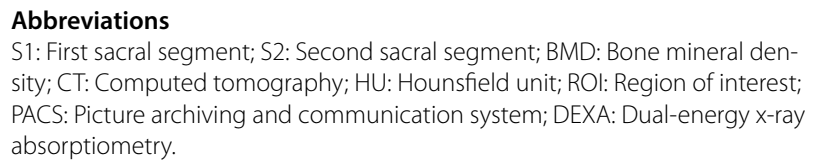
sity; CT: Computed tomography; HU: Hounsfield unit; ROI: Region of interest; PACS: Picture archiving and communication system; DEXA: Dual-energy $x$-ray absorptiometry.

\section{Acknowledgements}

The authors thank Dr. Heidi Israel for her work on statistical analysis and Dr. Lynne Bianchi for her work on manuscript revision. This study was conducted at Saint Louis University Hospital.

\section{Authors' Contributions}

All authors contributed to the study. All authors read and approved the final manuscript.

\section{Funding}

Not applicable.

Availability of data and materials

Not applicable.

\section{Ethics approval and consent to participate}

This study was reviewed and approved by the Saint Louis University Institutional Review Board (IRB) to ensure that it complied with ethical research practices on human subjects.

\section{Consent for publication}

Based on the retrospective analysis, individual patient consent was waived in the IRB approval.

\section{Competing interests}

None of the authors have any conflicts of interest related to this work to disclose. Authors have no proprietary interests in materials described in the article.

\footnotetext{
Author details

${ }^{1}$ Department of Orthopaedic Surgery, University of Pittsburgh Medical Center-Hamot, 201 State Street, Erie, PA 16550, USA. ${ }^{2}$ Department of Orthopaedic Surgery, Saint Louis University Medical School, 3635 Vista Ave, Saint Louis, MO 63110, USA. ${ }^{3}$ Department of Radiology, Saint Louis University Medical School, 3635 Vista Ave, Saint Louis, MO 63110, USA. ${ }^{4}$ Department of Radiology, Mallinckrodt Institute of Radiology, Washington University, 510 S Kingshighway Blvd, Saint Louis, MO 63110, USA. ${ }^{5}$ Department of Orthopaedics, Loyola University, Chicago, 2160 S 1st Ave, Maywood, IL 60153, USA.
} 
Received: 4 September 2019 Accepted: 24 April 2020

Published online: 25 May 2020

\section{References}

1. Bishop JA, Routt ML Jr (2012) Osseous fixation pathways in pelvic and acetabular fracture surgery: osteology, radiology, and clinical applications. J Trauma Acute Care Surg 72(6):1502-1509

2. Shuler TE, Boone DC, Gruen GS et al (1995) Percutaneous iliosacral screw fixation: early treatment for unstable posterior pelvic ring disruptions. J Trauma 38:453-458

3. Matta JM, Tornetta P 3rd (1996) Internal fixation of unstable pelvic ring injuries. Clin Orthop Relat Res 329:129-140

4. Keating JF, Werier J, Blachut P et al (1999) Early fixation of the vertically unstable pelvis: the role of iliosacral screw fixation of the posterior lesion. J Orthop Trauma 13:107-113

5. Routt ML Jr, Simonian PT (1996) Closed reduction and percutaneous skeletal fixation of sacral fractures. Clin Orthop Relat Res 329:121-128

6. Moed BR, Geer BL (2006) S2 iliosacral screw fixation for disruptions of the posterior pelvic ring: a report of 49 cases. J Orthop Trauma 20:378-383

7. Cole JD, Blum DA, Ansel LJ (1996) Outcome after fixation of unstable posterior pelvic ring injuries. Clin Orthop Relat Res 329:160-179

8. Matta JM, Saucedo T (1989) Internal fixation of pelvic ring fractures. Clin Orthop Relat Res 83-97

9. Carlson DA, Scheid DK, Maar DC et al (2000) Safe placement of S1 and S2 iliosacral screws: the "vestibule" concept. J Orthop Trauma 14:264-269

10. Arman C, Naderi S, Kiray A et al (2009) The human sacrum and safe approaches for screw placement. J Clin Neurosci 16:1046-1049

11. Hinsche AF, Giannoudis PV, Smith RM (2002) Fluoroscopy based multiplanar image guidance for insertion of sacroiliac screws. Clin Orthop Relat Res 395:135-144

12. Tonetti J, Cloppet O, Clerc M et al (2000) Implantation of iliosacral screws. Simulation of optimal placement by 3 -dimensional X-ray computed tomography. Revue de chirurgie orthopedique et reparatrice de l'appareil moteur 86:360-369

13. Ziran BH, Smith WR, Towers J et al (2003) Iliosacral screw fixation of the posterior pelvic ring using local anaesthesia and computerised tomography. J Bone Joint Surg Br 85:411-418

14. Nottmeier EW, Pirris SM, Balseiro S et al (2010) Three-dimensional imageguided placement of S2 alar screws to adjunct or salvage lumbosacral fixation. Spine J 10:595-601

15. Conflitti JM, Graves ML, Chip Routt ML Jr (2010) Radiographic quantification and analysis of dysmorphic upper sacral osseous anatomy and associated iliosacral screw insertions. J Orthop Trauma 24:630-636
16. Miller AN, Routt ML Jr (2012) Variations in sacral morphology and implications for iliosacral screw fixation. J Am Acad Orthop Surg 20(1):8-16

17. Gardner MJ, Morshed S, Nork SE et al (2010) Quantification of the upper and second sacral segment safe zones in normal and dysmorphic sacra. J Orthop Trauma 24:622-629

18. Kaiser SP, Gardner MJ, Liu J et al (2014) Anatomic determinants of sacral dysmorphism and implications for safe iliosacral screw placement. J Bone Joint Surg Am 96(14):e120(1-8)

19. Zou D, LiW, Xu F et al (2019) Use of Hounsfield units of S1 body to diagnose osteoporosis in patients with lumbar degenerative diseases. Neurosurg Focus 46(5):E6

20. Salazar D, Lannon S, Pasternak O et al (2015) Investigation of bone quality of the first and second sacral segments amongst trauma patients: concerns about iliosacral screw fixation. J Orthop Traumatol 16(4):301-308

21. Griffin DR, Starr AJ, Reinert CM et al (2006) Vertically unstable pelvic fractures fixed with percutaneous iliosacral screws: does posterior injury pattern predict fixation failure? J Orthop Trauma 20:S30-S36

22. van Zwienen CM, van den Bosch EW, Snijders CJ et al (2004) Biomechanical comparison of sacroiliac screw techniques for unstable pelvic ring fractures. J Orthop Trauma 18:589-595

23. Yinger K, Scalise J, Olson SA et al (2003) Biomechanical comparison of posterior pelvic ring fixation. J Orthop Trauma 17:481-487

24. Grampp S, Genant HK, Mathur A et al (1997) Comparisons of noninvasive bone mineral measurements in assessing age-related loss, fracture discrimination, and diagnostic classification. J Bone Miner Res 12:697-711

25. Schreiber JJ, Anderson PA, Rosas HG et al (2011) Hounsfield units for assessing bone mineral density and strength: a tool for osteoporosis management. J Bone Joint Surg Am 93:1057-1063

26. Donohue D, Decker S, Ford J et al (2018) Opportunistic CT screening for osteoporosis in patients with pelvic and acetabular trauma: technique and potential clinical impact. J Orthop Trauma 32(8):408-413

27. Wagner SC, Formby PM, Helgeson MD et al (2016) Diagnosing the undiagnosed: osteoporosis in patients undergoing lumbar fusion. Spine (Phila Pa 1976) 41(21):E1279-E1283

28. Pickhardt PJ, Pooler BD, Lauder T et al (2013) Opportunistic screening for osteoporosis using abdominal computed tomography scans obtained for other indications. Ann Intern Med 158(8):588-595

29. Hoel RJ, Ledonio CG, Takahashi T et al (2017) Sacral bone mineral density (BMD) assessment using opportunistic CT scans. J Orthop Res 35(1):160-166

\section{Publisher's Note}

Springer Nature remains neutral with regard to jurisdictional claims in published maps and institutional affiliations.

\section{Submit your manuscript to a SpringerOpen ${ }^{\circ}$ journal and benefit from:}

- Convenient online submission

- Rigorous peer review

- Open access: articles freely available online

- High visibility within the field

- Retaining the copyright to your article

Submit your next manuscript at $\boldsymbol{\nabla}$ springeropen.com 